\title{
The High Energy Particle Detector (HEPD-02) for the second China Seismo-Electromagnetic Satellite (CSES-02)
}

\section{Cristian De Santis $^{a, *}$ and Sergio Bruno Ricciarini ${ }^{b}$ on behalf of the CSES-Limadou Collaboration}

(a complete list of authors can be found at the end of the proceedings)

a INFN Sezione di Roma Tor Vergata,

Via della Ricerca Scientifica 1, I-00013 Rome, Italy

${ }^{b}$ IFAC-CNR,

Via Madonna del Piano, 10, I-50019 Sesto Fiorentino (Florence), Italy

E-mail: desantis@roma2.infn.it, s.ricciarini@ifac.cnr.it

CSES (China Seismo-Electromagnetic Satellite) is a multi-instrumental scientific space program whose objectives are to investigate the near-Earth electromagnetic, plasma and particle environment and to study the seismo associated disturbances in the ionosphere-magnetosphere transition zone, the anthropogenic electromagnetic noise as well as the natural non-seismic electromagnetic emissions, mainly due to tropospheric activity. In particular, the mission aims at confirming the existence of possible temporal correlations between the occurrence of medium and strong magnitude earthquakes and the observation in space of electromagnetic perturbations, plasma variations and precipitation of bursts of high-energy charged particles from the inner Van Allen belt.

The first satellite (CSES-01) was launched on 2018, while a second one (CSES-02) is currently under development and its launch is expected by the end of 2022.

As in CSES-01, the suite of instruments on-board CSES-02 will comprise a particle detector (HEPD-02, High-energy Particle Detector) to measure the increase of the electron and proton fluxes due to short-time perturbations of the radiation belts induced by solar, terrestrial or anthropic phenomena in the energy range 3-100 MeV for electrons and 30-200 MeV for protons.

HEPD-02 comprises a tracker made of CMOS Monolithic Active Pixel Sensors (MAPS), a double layer of crossed plastic scintillators for trigger and a calorimeter, made of a tower of plastic scintillators and a matrix of inorganic crystals, surrounded by plastic scintillator planes for containment tagging.

The main characteristics and performance of HEPD-02 are presented, highlighting the architectural choices made to meet the scientific objectives of the mission.

$37^{\text {th }}$ International Cosmic Ray Conference (ICRC 2021)

July 12 th - 23rd, 2021

Online - Berlin, Germany

\footnotetext{
${ }^{*}$ Presenter
} 


\section{Introduction}

CSES (China Seismo-Electromagnetic Satellite)[1] is a scientific space program which foresees the development of a multi-satellite monitoring system including several missions scheduled for the next years. The first satellite (CSES-01) was launched on 2018 while the second one (CSES-02) is currently under development and it is expected to be launched by the end of 2022 .

The Chinese-Italian CSES missions are dedicated to the monitoring of variations in the electromagnetic field and waves, plasma parameters and Van Allen belt particle fluxes, induced by natural sources and artificial emitters in the near-Earth space. One major goal is to investigate possible temporal correlations between such perturbations and the occurrence of high-magnitude seismic events. Other fundamental targets are the study of space-weather phenomena $[2,3]$ and cosmic-ray propagation [4].

CSES-02, quite similar to CSES-01, is based on the 3-axis-stabilized CAST2000 platform (total mass $900 \mathrm{~kg}$, peak power consumption $900 \mathrm{~W}$, storage $512 \mathrm{~GB}$ ) developed by DFH Satellite Co., Ltd.; it will fly on a sun-synchronous polar orbit at $\sim 507 \mathrm{~km}$ altitude, with $97^{\circ}$ inclination, a period of $94.6 \mathrm{~min}$, and a 5-day revisiting period. CSES-02 will follow a complementary ground track with respect to CSES-01 (identical orbit plane, with $180^{\circ}$ phase difference) in order to maximize the observation capabilities.

A suite of ten scientific payloads, with a few minor differences respect CSES-01, is housed inside the satellite: two particle detectors, namely a Low Energy Electron Spectrometer and a High-Energy Particle Detector (HEPD-02); a High-Precision Magnetometer (HPM); a Search-Coil Magnetometer (SCM); an Electric Field Detector (EFD-02); a Global Navigation Satellite System (GNSS) Occultation Receiver; a Tri-Band Beacon transmitter (TBB); a Langmuir Probe (LAP); a Plasma Analyzer Package (PAP); an Ionospheric Photometer. Unlike CSES-01, payloads will work along the whole orbit.

The Italian branch of the CSES collaboration, named Limadou, has been developing HEPD-02 and EFD-02.

\section{HEPD-02}

The High Energy Particle Detector (HEPD-02) is capable of detecting individual incident particles, identifying the type (proton, electron, nucleus), measuring the energy and determining the angle between the flight line and the Earth's magnetic field line to the Equator (pitch angle). The main purpose is to identify particle flows ("particle burst") suddenly falling from the stability bands of the Van Allen internal belt into the atmosphere and to determine with great accuracy their magnetospheric region of origin and the energy spectrum, with the aim to find possible temporal correlations between the bursts and terrestrial seismic events.

HEPD-02 consists of five subsystems: Detector (DES), Mechanics (MES), Electronics (ELS), Power-Supply (PSS), Harness (HAS). The main general and technical requirements for HEPD-02 are reported in Table 1.

\subsection{HEPD-02 Detector}

The Detector Subsystem (DES) consists of the following sub-detectors: 


\begin{tabular}{|l|r|}
\hline Operating temperature & $-10^{\circ} \mathrm{C} \div+35^{\circ} \mathrm{C}$ \\
\hline Operating pressure & $\leq 6.65 \cdot 10^{-3} \mathrm{~Pa}$ \\
\hline Data budget & $\leq 100 \mathrm{~Gb} / \mathrm{day}$ \\
\hline Mass budget & $\leq 50 \mathrm{~kg}$ \\
\hline Power budget & $\leq 45 \mathrm{~W}$ \\
\hline Electron kinetic energy range & $3 \mathrm{MeV} \div 100 \mathrm{MeV}$ \\
\hline Proton kinetic energy range & $30 \mathrm{MeV} \div 200 \mathrm{MeV}$ \\
\hline Angular resolution & $\leq 10^{\circ}$ for $e^{-}$with E $>3 \mathrm{MeV}$ \\
\hline Energy resolution & $\leq 10 \%$ for $e^{-}$with E $>5 \mathrm{MeV}$ \\
\hline Pointing & Zenith \\
\hline Scientific data bus & RS-422 \\
\hline Data handling bus & CAN 2.0 \\
\hline Life cycle & $>6$ years \\
\hline
\end{tabular}

Table 1: HEPD-02 main requirements.

- Direction Detector (DD);

- Trigger Detector (TD);

- Energy Detector (ED);

- Containment Detector (CD).

An overall view of the detector layout is shown in Figure 1a and Figure 1b. The detailed design of the detector is presented along with the architectural choices made to meet the scientific objectives of the mission.

\subsubsection{Direction Detector (DD)}

The Direction Detector (DD) measures the entrance angle of the particle and it is based on the ALPIDE Monolithic Active Pixel Sensor (MAPS), a $15 \times 30 \mathrm{~mm}^{2}$ chip designed at CERN for ALICE Inner Tracker System (ITS) Upgrade and implemented in $180 \mathrm{~nm}$ CMOS Imaging Process technology on high resistivity p-doped silicon wafer of $50 \mu \mathrm{m}$ thickness.

The DD consists of five standalone turrets $\left(225 \times 38 \times 12 \mathrm{~mm}^{3}\right)$, each made of three stacked sensitive planes (or "staves"). This choice is a trade-off between segmentation into independent sections and increase of power, dead time and cabling. The sensitive area of each turret's plane consists of $2 \times 5=10$ ALPIDE sensors, each containing $1024 \times 512$ almost square pixels of about $28 \mu \mathrm{m}$ side. The sensors are glued on a CFRP Cold Plate (CP) and connected to a superimposed Flexible Printed Circuit (FPC) via wire bondings. The external electrical interface is made by a tracker splitter (TSP) board per turret, which is installed on the support mechanics of the DD.

\subsubsection{Trigger Detector (TD)}

The Trigger Detector (TD) consists of two rectangular EJ-200 plastic scintillator planes, TR1 and TR2, segmented in bars read-out by one Hamamatsu R9880-210 PhotoMultiplier Tube (PMT) 


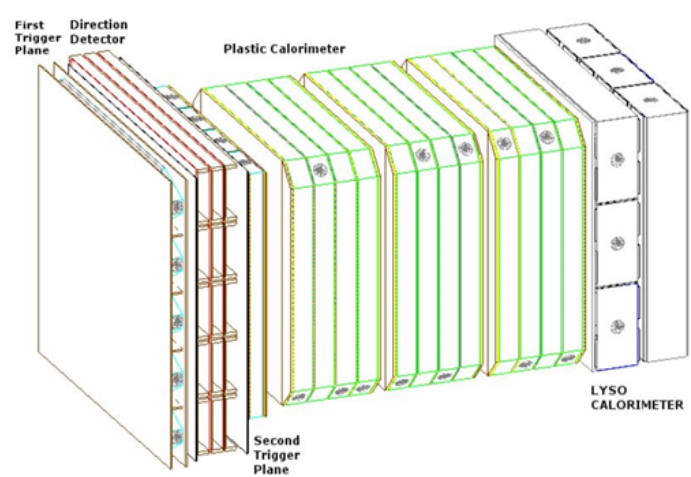

(a) HEPD-02 detector layout.

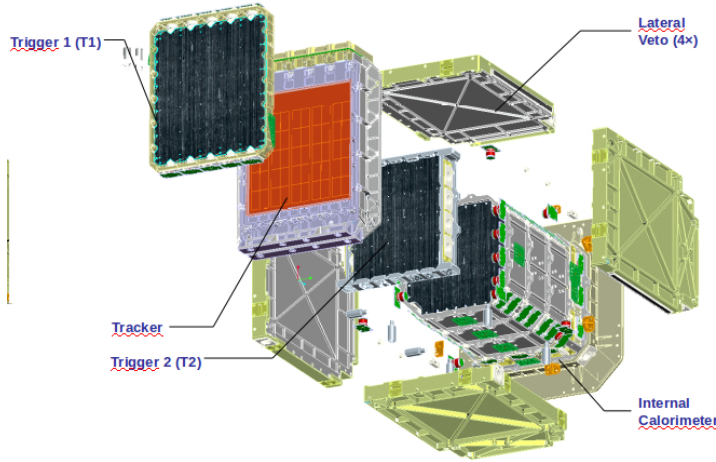

(b) HEPD-02 detector details.

Figure 1: Left panel: HEPD-02 detector layout. Right panel: Exploded view of the HEPD-02 Detector Subsystem (DES) with details of its sub-detectors.

at each end. The two planes are segmented in bars along orthogonal directions ( $\mathrm{Y}$ and $\mathrm{X}$ axis respectively) and their horizontal (XY) dimensions have been defined in such a way that triggered events from both TR1 and TR2 planes mostly have incoming direction pointing toward the inner part of the underlying ED layers.

The first trigger plane, TR1, is set on top of the Direction Detector. The overall dimensions of TR1 are $154.6 \times 162.5 \times 2 \mathrm{~mm}^{3}$; it is segmented in 5 scintillator bars (dimensions $154.6 \times 32.5 \times 2$ $\mathrm{mm}^{3}$ each) along the $\mathrm{Y}$ axis, corresponding to the $5 \mathrm{DD}$ turrets. Furthermore, the overall material budget of the scintillator and support material has been minimized to reduce the amount of particle interactions before the measurement performed by the DD. Specifically, the horizontal and vertical internal support walls are made of light CFRP composite instead of aluminum alloy; this same design rule has been adopted for TR2 and RAN detectors, discussed in what follows.

For TR1, light is collected on the PMT by using a light guide. The use of light guides in TR1 is necessary to maximize the geometric light collection efficiency on the circular ( $8 \mathrm{~mm}$ diameter) optical window of the PMT, given the rectangular $32.5 \times 2 \mathrm{~mm}^{2}$ area of the counter end side. Light guides on the two ends are not symmetric, thus allowing for an extremely tight vertical packing of TR1 and DD with the corresponding support mechanics, which in turn results in minimizing triggers from TR1 when the incoming particle does not cross the active area of the underlying ALPIDE staves.

The second trigger plane TR2 is set in between the DD and the underlying ED. The thickness of TR2 scintillators has been optimize to satisfy the mechanical requirements and to deliver a high enough number of photons and, consequently, an acceptable event-by-event fluctuation of the signal from traversing charged particles. TR2 is segmented into 4 bars along the $\mathrm{X}$ axis; the overall dimensions of each TR2 bar are $36 \times 150 \times 8 \mathrm{~mm}^{3}$. The horizontal $(\mathrm{XY})$ dimensions of the envelope of the scintillator bars match the ones of the underlying ED planes.

\subsubsection{Energy Detector (ED)}

The Energy Detector (ED) is a calorimeter consisting of a tower with twelve EJ-200 plastic scintillator planes on top of two layers of crystal (LYSO) scintillators bars. The plastic scintillator 
planes are collectively indicated as RAN ("range measurement") layers, while the LYSO scintillator bars are collectively indicated as EN ("energy measurement") layers. The amount of employed LYSO material is limited by the mass budget of HEPD-02; furthermore, the overall linear thickness of the ED sensitive layers (plus the TD layers, contributing also to the energy measurement) is mainly limited by the allowed geometrical dimensions of the HEPD-02 payload.

Each RAN plastic scintillator plane measures $150 \times 150 \times 10 \mathrm{~mm}^{3}$ at the envelope (excluding mechanics); it is read-out by two Hamamatsu R9880-210 PMTs located at opposite corners. The RAN support structure is made of three identical mechanical modules, each one containing 4 scintillator planes with interleaved thin elastomer layers.

The EN section is formed by two planes, each one measuring $150 \times 150 \times 25 \mathrm{~mm}^{3}$ at the envelope (excluding mechanics); each plane is segmented into three $150 \times 49 \times 25 \mathrm{~mm}^{3}$ LYSO bars, with bars of the first plane orthogonal to those of the second. Each bar is read-out by 2 PMTs located at the opposite ends, in similar way to the TR2 bar.

\subsubsection{Containment Detector (CD)}

The Containment Detector (CD) consists of 5 plastic scintillator panels: four LAT (lateral) panels and one BOT (bottom) panel, located at the bottom of the instrument; each panel is $8 \mathrm{~mm}$ thick and read-out by two Hamamatsu R9880-210 PMTs located at opposite corners. The LAT panels cover from TR2 down to the BOT panel, thus allowing to tag low-quality categories of events, such as those with particles entering the ED from lateral or bottom sides (whose signal contribution adds to the proper signal from the particle crossing the TD and giving the trigger signal) or those with particles not stopping inside the ED and thus escaping the ED lateral or bottom surfaces (the energy measurement for these particles is generally affected by a significant missing fraction).

\subsection{HEPD-02 Performance}

HEPD-02 has been designed to meet the scientific requirements reported in Table 1, with particular attention to the electron and proton angular and energy resolution in the explored energy range. Given the demanding mechanical constraints, the detector has been carefully studied to obtain an optimal trade-off between active materials and support structures along the vertical axis. The scientific performance has been evaluated by means of a Geant 4 simulation for an isotropic incoming flux of electrons and protons on top of the instrument.

The expected detectable energy ranges for electrons and protons are shown in Figure 2. The mission requirement (3 to $100 \mathrm{MeV}$ electrons, 30 to $200 \mathrm{MeV}$ protons) is met for both particle species.

The expected energy resolution is illustrated in Figure 3: the electron energy uncertainty is foreseen to be less than $10 \%$ for kinetic energies $>5 \mathrm{MeV}$, in compliance with the mission requirement.

The expected angular resolution of the detector is reported in Figure 4. The angular resolution turns out to be better than $10^{\circ}$ for the larger part of the electron events with kinetic energies above $5 \mathrm{MeV}$, thus satisfying the corresponding requirement. 


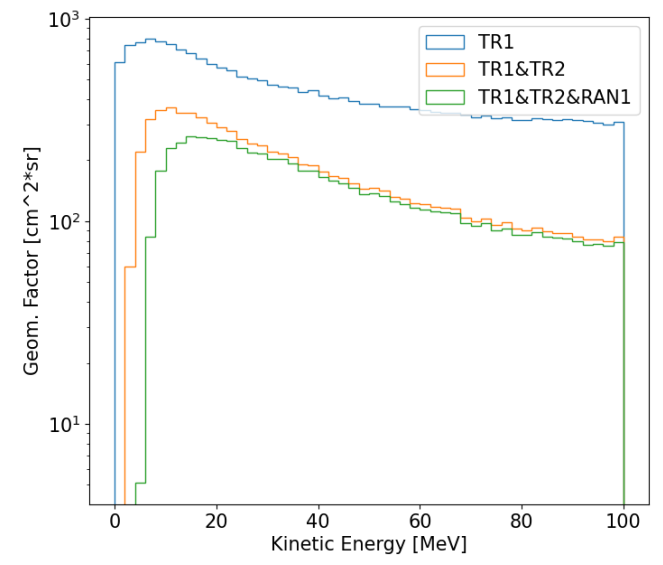

(a) Electron geometric factor.

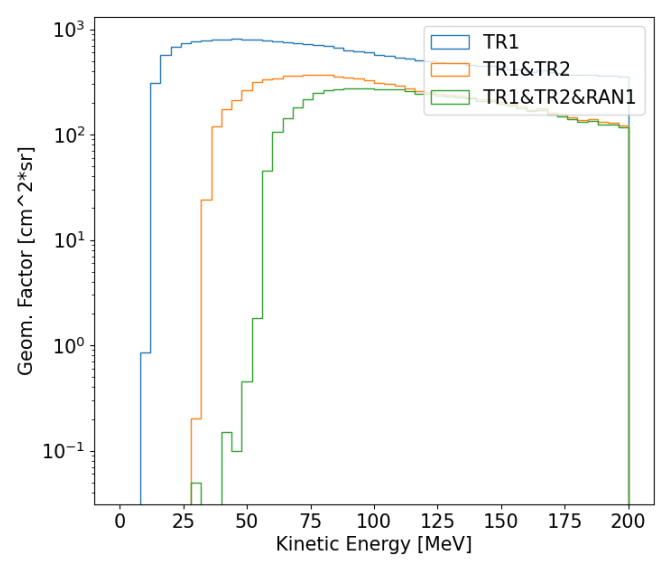

(b) Proton geometric factor.

Figure 2: Simulation of HEPD-02 geometric factor for electrons (left) and protons (right), by requiring the production of a trigger signal in different trigger configurations.

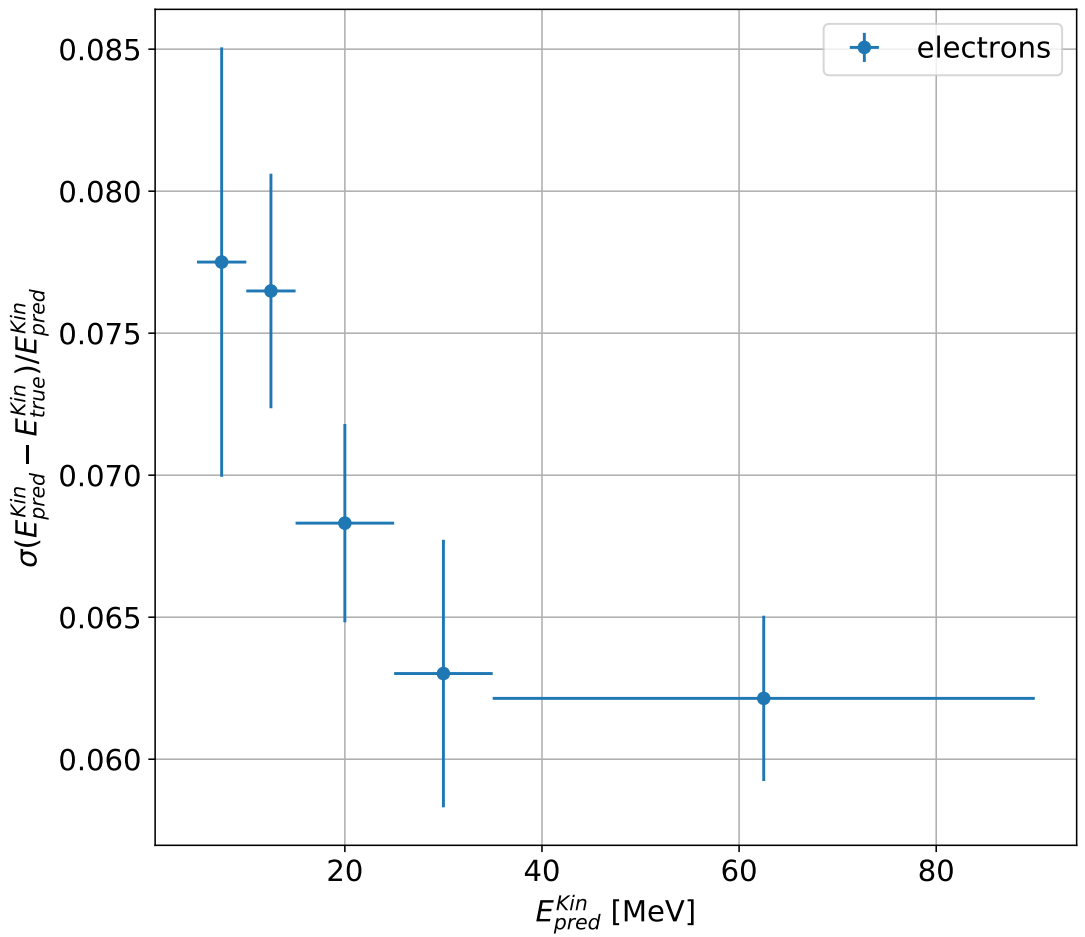

Figure 3: Simulation of the relative difference between initial kinetic energy and reconstructed one, for a sample of electrons satisfying typical event selection criteria applied to remove background and out-ofacceptance events. 


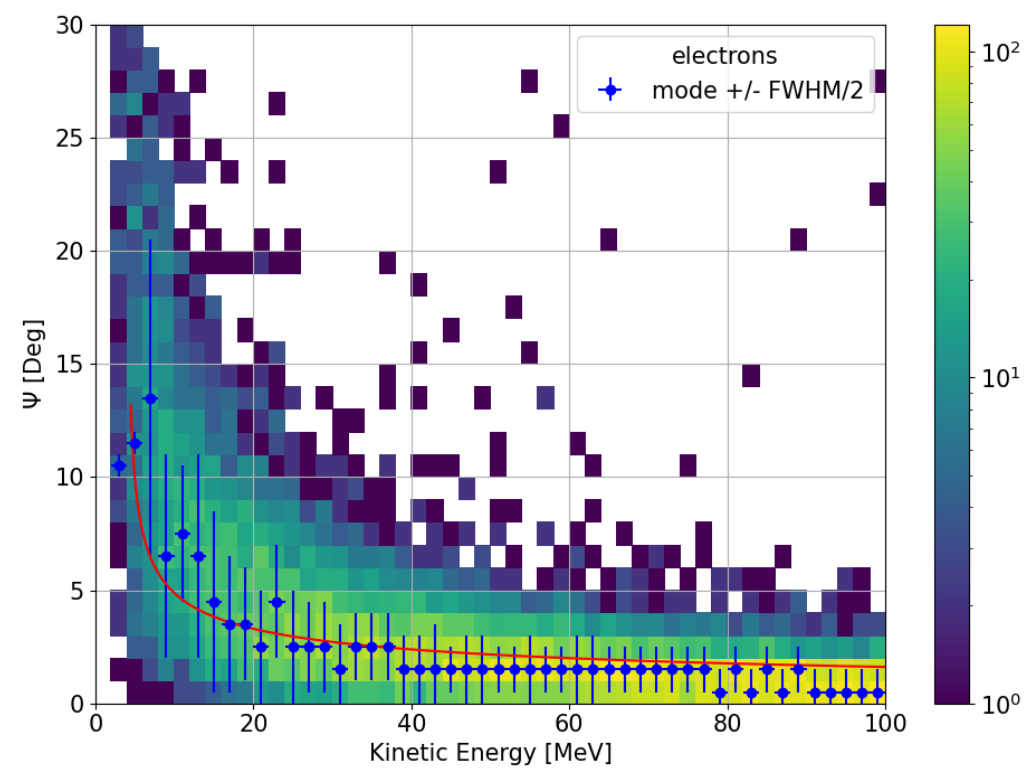

Figure 4: Simulated distribution of the difference angle $\Psi$ between incoming electron direction as reconstructed in the DD and the true one, for a sample of electrons satisfying typical event selection criteria applied to remove background and out-of-acceptance events.

\section{Conclusions}

In this contribution we present the design and simulated performances of the High Energy Particle Detector (HEPD-02), which is currently being developed and expected to be launched on-board of the second China Seismo-Electromagnetic Satellite (CSES-02) by the end of 2022. The detector architecture is the result of an optimized trade-off between scientific objectives of the mission and tight technical requirements for high-reliability operation in space environment.

\section{References}

[1] Shen, X. et al., The state-of-art of the China Seismo-Electromagnetic Satellite mission, Science China Technological Sciences, vol. 61, no. 5, pp.634-642, May 2018.

[2] Zhima, Z. et al., Storm-Time Features of the Ionospheric ELF/VLF Waves and Energetic Electron Fluxes Revealed by the China Seismo-Electromagnetic Satellite, Appl. Sci. 2021, 11, 2617.

[3] Yang, Y.-Y. et al., The First Intense Storm Event Recorded by the China SeismoElectromagnetic Satellite, Space Weather 2019, 18 (1).

[4] Bartocci, S. et al., Galactic Cosmic-Ray Hydrogen Spectra in the 40-250 MeV Range Measured by the High-energy Particle Detector (HEPD) on board the CSES-01 Satellite between 2018 and 2020, ApJ 2020, 901, 8. 


\section{Full Authors List: CSES-Limadou Collaboration}

S. Bartocci ${ }^{1}$, R. Battiston ${ }^{2,3}$, F. Benotto ${ }^{4}$, S. Beole ${ }^{4,5}$, W.J. Burger ${ }^{3,6}$, D. Campana ${ }^{7}$, G. Castellini ${ }^{8}$, P. Cipollone ${ }^{1}$, S. Coli ${ }^{4}$, L. Conti ${ }^{1,9}$, A. Contin ${ }^{10,11}$, M. Cristoforetti ${ }^{12}$, L. De Cilladi ${ }^{4,5}$, C. De Donato ${ }^{1}$, C. De Santis ${ }^{1}$, F.M. Follega ${ }^{2,3}$, G. Gebbia ${ }^{2,3}$, R. Iuppa ${ }^{2,3}$, M. Lolli $^{11}$, N. Marcelli $1{ }^{1,13}$, M. Martucci ${ }^{1,13}$, G. Masciantonio ${ }^{1}$, M. Mergé ${ }^{1, \dagger}$, M. Mese ${ }^{7,14}$, C. Neubuser ${ }^{3}$, F. Nozzoli ${ }^{3}$, A. Oliva ${ }^{11}$, G. Osteria $^{7}$, L. Pacini ${ }^{15}$, F. Palma ${ }^{1, \dagger}$, F. Palmonari ${ }^{10,11}$, A. Parmentier ${ }^{1}$, F. Perfetto ${ }^{7}$, P. Picozza ${ }^{1,13}$, M. Piersanti ${ }^{16}$, M. Pozzato ${ }^{11}$, E.

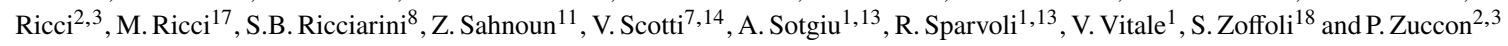

${ }^{1}$ INFN-Sezione di Roma “Tor Vergata”, V. della Ricerca Scientifica 1, I-00133 Rome, Italy;

${ }^{2}$ University of Trento, V. Sommarive 14, I-38123 Povo (Trento), Italy;

${ }^{3}$ INFN-TIFPA, V. Sommarive 14, I-38123 Povo (Trento), Italy;

${ }^{4}$ INFN-Sezione di Torino, Via P. Giuria 1, I-10125 Torino, Italy;

${ }^{5}$ University of Torino, Via P. Giuria 1, I-10125 Torino, Italy;

${ }^{6}$ Centro Fermi, V. Panisperna 89a, I-00184 Rome, Italy;

${ }^{7}$ INFN-Sezione di Napoli, V. Cintia, I-80126 Naples, Italy;

${ }^{8}$ IFAC-CNR, V. Madonna del Piano 10, I-50019 Sesto Fiorentino (Florence), Italy;

${ }^{9}$ Uninettuno University, C.so V. Emanuele II 39, I-00186 Rome, Italy;

${ }^{10}$ University of Bologna, V.le C. Berti Pichat 6/2, I-40127 Bologna, Italy;

${ }^{11}$ INFN-Sezione di Bologna, V.le C. Berti Pichat 6/2, I-40127 Bologna, Italy;

${ }^{12}$ Fondazione Bruno Kessler, V. Sommarive 18, I-38123 Povo (Trento), Italy;

${ }^{13}$ University of Rome "Tor Vergata", V. della Ricerca Scientifica 1, I-00133 Rome, Italy;

${ }^{14}$ University of Naples "Federico II", V. Cintia 21, I-80126 Naples, Italy;

${ }^{15}$ INFN-Sezione di Firenze, V. Sansone 1, I-50019 Sesto Fiorentino (Florence), Italy;

${ }^{16}$ INAF-IAPS, V. Fosso del Cavaliere 100, I-00133 Rome, Italy;

${ }^{17}$ INFN-LNF, V. E. Fermi 54, I-00044 Frascati (Rome), Italy;

${ }^{18}$ Italian Space Agency, V. del Politecnico, I-00133 Rome, Italy;

$\dagger$ At ASI Space Science Data Center (SSDC) also, V. del Politecnico, I-00133 Rome, Italy. 